\title{
ETNOBOTÁNICA Y ECOLOGÍA DE PLANTAS UTILIZADAS POR TRES CURANDEROS CONTRA LA MORDEDURA DE SERPIENTE EN LA REGIÓN DE ACAYUCAN, VeraCRUZ, MÉXICO
}

\author{
Mario Ramos-Hernández', Carlos H. Ávila-Bello ${ }^{1,3}$ y Jorge E. Morales-Mávil ${ }^{2}$ \\ 'Facultad de Ingeniería en Sistemas de Producción Agropecuaria, Universidad Veracruzana. \\ Km 4.5 Carretera Costera del Golfo Acayucan-Catemaco, Acayucan 96000, Veracruz, México. \\ ${ }^{2}$ Instituto de Neuroetología. Universidad Veracruzana, Xalapa 91000,Veracruz, México. \\ ${ }^{3}$ Autor para la correspondencia. Correo-e: carlavila@uv.mx
}

\begin{abstract}
Resumen: Se estudió el conocimiento etnobotánico y se analizaron aspectos ecológicos de plantas utilizadas como antiviperino por tres curanderos de la región de Acayucan, Veracruz, México. Los objetivos fueron conocer las plantas usadas para la elaboración del suero y evaluar la estructura general de la vegetación en dos áreas en donde se recolectan estas plantas. Se llevaron a cabo entrevistas y recorridos de campo con los tres curanderos, se calcularon las frecuencias relativas de las especies y se registró su forma de uso. La estructura de la vegetación se estudió en sitios de $400 \mathrm{~m}^{2}$ divididos en parcelas de $25 \mathrm{~m}^{2}$. Se determinaron 16 especies usadas contra la mordedura de serpiente. Las plantas se preparan de tres formas: cocimiento, pulverización y extracto alcohólico; todos los métodos se aplican vía oral. Las zonas de recolección son áreas muy aisladas y fragmentadas, rodeadas por terrenos utilizados para la agricultura y la ganadería.
\end{abstract}

Palabras clave: antiviperino, conocimiento tradicional, estructura de la vegetación, Veracruz.

\begin{abstract}
We studied the ethnobotanical knowledge and analyzed some ecological aspects of plants used in treatments against snakebite by three traditional healers in the Acayucan region, Veracruz, Mexico. The objectives were to identify those plants used in the preparation of the antidote and to describe the overall vegetation structure of two sites where the healers collect these plants. The three healers were interviewed, the relative frequencies of species used were calculated, and the form of use were recorded. Vegetation structure in areas of plant collection was studied by means of $400 \mathrm{~m}^{2}$-sites, divided into $2525 \mathrm{~m}$ plots. We recorded 16 species that are prepared in three different ways, namely cooking, pulverization and alcoholic extract; all of them are administered orally. The sites where plants are collected are very isolated and fragmented, as they are surrounded by areas used for agriculture and cattle ranching.
\end{abstract}

Key words: antiviperin, traditional knowledge, vegetation structure, Veracruz.

$\mathbf{L}$ a etnobotánica estudia las relaciones entre las plantas y los humanos. Estas relaciones han existido desde el momento en que el hombre inició el uso de los vegetales para satisfacer sus necesidades de supervivencia, ya sea como alimento, para producir calor, para abrigarse, en la construcción, como ornamento y para procurar su salud (Levy y Aguirre, 1999). A lo largo de este proceso, el ser humano adquirió conocimientos detallados de la localización y las características estructurales de la vegetación que aprovechaba. Para la etnobotánica, documentar el uso de las plantas medicinales, en particular las utilizadas como antiviperino, reviste especial interés por su potencial aplicación farmacológica. Del mismo modo, se busca contribuir al uso sustentable de los recursos naturales asociados a esta actividad, y cuantificarlos e inventariarlos es un primer paso para lograr este propósito (Tuxill y Nabhan, 1998; Martin, 2000), especialmente en el caso de áreas forestales en donde se recolectan diferentes especies.

El número de plantas superiores reportadas en la literatura internacional como antídoto contra la mordedura de 
serpientes comprende 578 especies distribuidas en 94 familias (Mors, 1991). En varias regiones del mundo, especialistas en la materia consideran que una de cada siete especies vegetales posee alguna propiedad curativa. Además, se estima que en México y el resto del mundo, la validación químico-farmacológica y biomédica sólo se ha llevado a cabo en 5\% de estas especies (Huerta, 2002). Los estudios realizados en países sudamericanos y en México con plantas antiviperinas muestran que los antídotos herbolarios pueden ser efectivos para contrarrestar algunos efectos del veneno como son edemas, mionecrosis, desfibrinación y coagulación (Nakawa y Nakanishi, 1982; Reyes-Chilpa et al., 1994; Pereira et al., 1994; Lans et al., 2001; Núñez et al., 2004).

Algunas de las plantas más ampliamente utilizadas en Brasil, Colombia y México para neutralizar los efectos del veneno de serpientes pertenecen a los géneros Mikania y Aristolochia, las cuales particularmente han sido probadas con ofidios del género Bothrops, aunque con otras plantas se han tenido resultados bastante halagadores (Mors, 1991; Ruppelt et al., 1991; Pereira et al., 1994; Robinson y López, 1999). Por ejemplo, Bixa orellana L. y Heliconia curtispatha Petersen inhiben entre 72 y $73 \%$ la formación de edemas en ratones inyectados con veneno de Bothrops asper. En cuanto a la desfibrinación, se requieren $3.9 \mu \mathrm{g}$ y $250 \mu \mathrm{g}$ de extracto por ratón, respectivamente, para neutralizarla totalmente (Nuñez et al., 2004). Por su parte, Bursera simaruba (L.) Sarg., Persea americana Mill. y Croton draco Schltdl. contienen gran cantidad de taninos condensados, lo que les permite inhibir los efectos neurotóxicos y hematotóxicos del veneno de esa misma especie (Castro et al., 1999). Dos plantas evaluadas en México, Brongniartia podalyrioides Kunth y B. intermedia Moric., contienen edunol, sustancia cuya estructura química le permite neutralizar los efectos cardiotóxicos del veneno (Reyes-Chilpa et al., 1994). Así mismo, los coras en Nayarit utilizan Salvia misella Kunth macerada y mezclada con alcohol u orina humana, aplicada como emplasto y por vía oral, para tratar la mordedura de serpientes ponzoñosas (Gispert-Cruells y Rodríguez-González, 1998). Según Mors (1991), Eclipta prostrata (L.) L. y Casearia sylvestris Sw. inhiben la acción miotóxica del veneno de las serpientes. El principio activo de E. prostrata es el sitosterol; además, esta planta también contiene estigmasterol que neutraliza los efectos hematotóxicos.

Existen estudios realizados con plantas medicinales útiles como antiviperinos en el estado de Veracruz, en los que se describen las formas de recolección, preparación y administración de dichos antídotos, así como las partes de las plantas que se utilizan (del Amo, 1979; Garrido, 1997). Sin embargo, la información sigue siendo escasa y adolece, en muchos casos, de un análisis ecológico relacionado con las características estructurales generales de las zonas donde estas especies son recolectadas. Aunado a lo anterior, el conocimiento tradicional relacionado con estas plantas se está perdiendo debido a la avanzada edad de los poseedores y al desinterés de los jóvenes por aprender el uso de las mismas.

Los objetivos del presente trabajo fueron: (1) conocer las plantas medicinales usadas como remedio antiviperino por tres curanderos de la región de Acayucan, Veracruz, (2) obtener información básica para documentar el conocimiento tradicional relacionado con esta actividad, y (3) describir las características estructurales generales de las áreas de colecta de estas plantas.

\section{Materiales y métodos}

Área de estudio. El estudio se desarrolló en las localidades El Serrano (1753’25” N y 95 $33^{\circ} 08^{\prime \prime}$ O; 70 m. s.n.m.), Rodríguez Clara (1759'31" N y 95'24'12" O, $130 \mathrm{~m}$. s.n.m.) y Sayula de Alemán (1752'54” N y 94²57’35” O, $80 \mathrm{~m}$ s.n.m.), todas ellas localizadas en el sureste de Veracruz, México. El Serrano forma parte del municipio de Playa Vicente, mientras que Rodríguez Clara y Sayula de Alemán son las cabeceras de sus respectivos municipios (figura 1).

El clima es cálido subhúmedo con lluvias en verano, con precipitación media anual de 2,000 mm para El Serrano, 1,500 mm para Rodríguez Clara y 1,650 mm para Sayula de Alemán. La temperatura media anual para las tres comunidades es de $26^{\circ} \mathrm{C}$. Los suelos son de tipo feozem en El Serrano, arenosoles en Rodríguez Clara y de tipo luvisol en Sayula de Alemán (INEGI, 2004).

El tipo de vegetación del área fue selva alta perennifolia. Actualmente se desarrolla agricultura de temporal con cultivos anuales y semipermanentes; sin embargo, domina el paisaje la cría de ganado bovino con pastizales cultivados (INEGI, 2004).

Recopilación y análisis de información etnobotánica. Para recabar la información etnobotánica, se identificó, por medio de recorridos de campo y entrevistas a miembros de las comunidades, a las personas que conservan el conocimiento acerca de las plantas medicinales. Aunque hay otras personas que realizan curaciones, se incluyó sólo a tres informantes clave, debido a que son quienes preparan los sueros antiviperinos y poseen mayor conocimiento al respecto, ya que tienen una estrecha relación con las plantas, conocen su fenología, sus usos y lugares de cosecha. Posteriormente se aplicaron, directamente en el campo, entre febrero y marzo de 2005, entrevistas estructuradas con base en preguntas directas (cuadro 1); esto permitió tener un mayor control sobre las respuestas, además de poder cuantificar y analizar estadísticamente los datos recolectados (Alexiades, 1996). Además, se recolectaron por duplicado ejemplares botánicos para su posterior identificación. Los especímenes se enviaron al herbario CIB del 


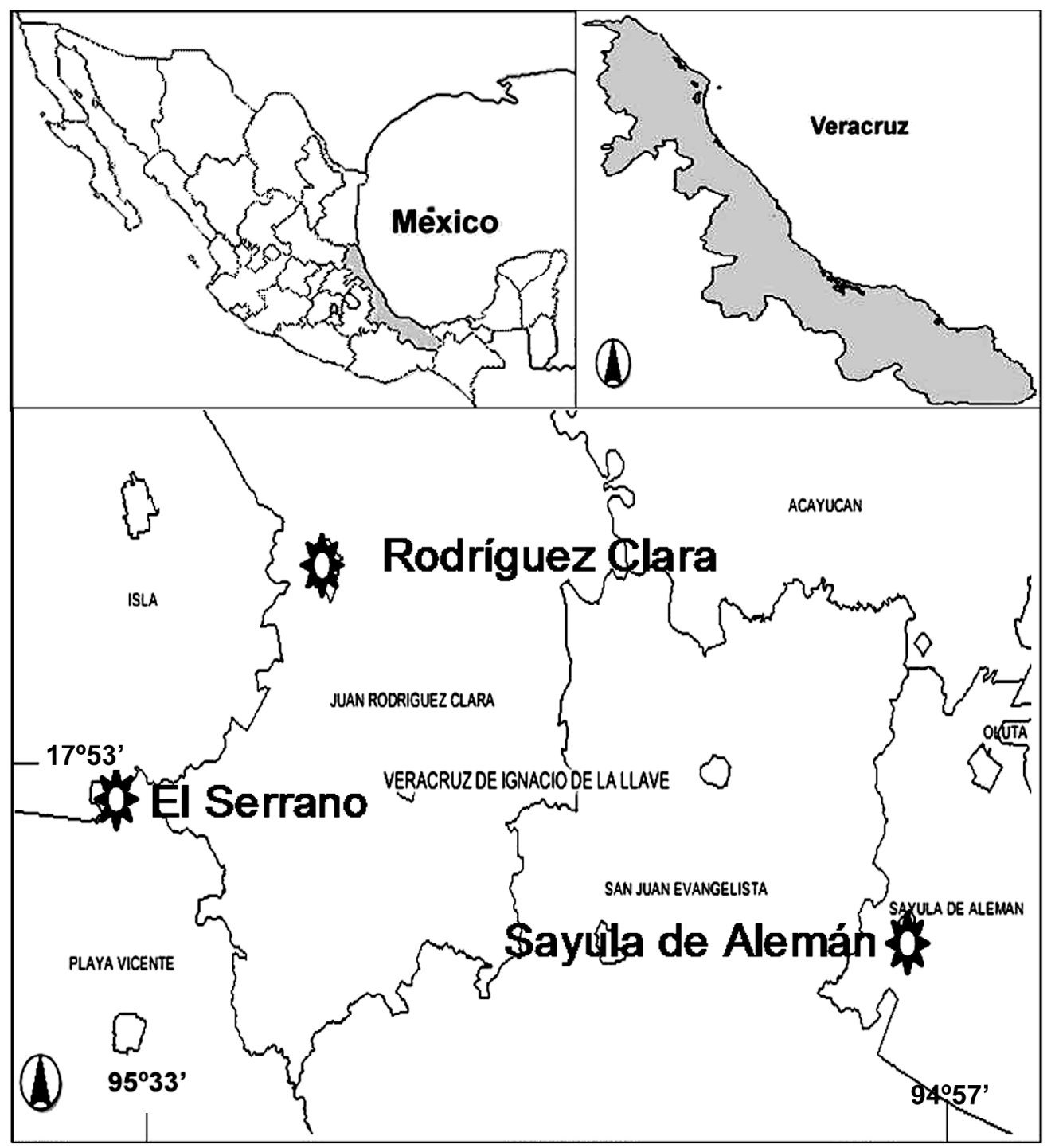

Figura 1. Localización de las tres localidades estudiadas, representadas por los puntos con marcas en forma de estrella (El Serrano, 1753' 25” N y 9533'08” O, altitud de 70 m s.n.m.), Rodríguez Clara (1759’31” N y 95²4'12” O, 130 m s.n.m.) y Sayula de Alemán (1752' 54” N y 9457’35”, 80 m s.n.m.) (INEGI, 2004).

Instituto de Investigaciones Biológicas de la Universidad Veracruzana en Xalapa, Veracruz; los números de registro preliminar de estas plantas se presentan en el cuadro 2 .

Recopilación y análisis de información ecológica. Los lugares en donde uno de los informantes recolecta las plantas para elaborar el suero antiviperino se encuentran muy fragmentados y perturbados por la influencia de la actividad ganadera. Sólo fue posible establecer dos sitios de 400 $\mathrm{m}^{2}$ ( $20 \mathrm{~m} \quad 20 \mathrm{~m}$ cada uno), los que se dividieron a su vez en subcuadros de $25 \mathrm{~m}^{2}(5 \mathrm{~m} 5 \mathrm{~m})$, en donde se obtuvieron los datos para estimar las características estructurales generales; se realizó un sorteo para elegir un subcuadro y obtener los datos del estrato herbáceo. Del estrato arbóreo se anotaron el diámetro a la altura del pecho (DAP; $1.3 \mathrm{~m}$ del suelo), la altura total, la altura del fuste y la cobertura. Con estos datos se obtuvo el área basal de los árboles, la densidad relativa, la dominancia relativa con base en el área basal y la frecuencia relativa de todas las especies arbóreas (Shimwell, 1972). Con estas variables se calculó el valor de importancia (V.I.) de las especies arbóreas; el V.I. permite determinar cuáles son las especies que dominan el ecosistema y contribuyen a asegurar la funcionalidad del mismo (Curtis y McIntosh, 1951; Curtis, 1959; Connell y Slatyer, 1977; Sánchez-Velázquez y Pineda-López, 2000). 
Cuadro 1. Cuestionario utilizado para obtener los datos etnobotánicos.

Nombre del entrevistado:

Sexo:

Edad:

Lugar y fecha de nacimiento:

Lugar donde vive actualmente:

Tiempo que lleva viviendo allí:

Fuente de economía u oficio:

Personas que dependen del entrevistado:

1) ¿Conoce algunas plantas utilizadas contra el veneno de serpientes?

2) ¿Puede decir cuáles son?

3) ¿Qué tipo de planta es? (árbol, arbusto, hierba, trepadora)

4) ¿Cuál de ellas es más abundante en la zona?

5) ¿Dónde se encuentran esas plantas?

6) ¿Cuál es la más utilizada en su comunidad y por qué?

7) ¿Qué parte de la planta se utiliza?

8) ¿Cuál es la forma de preparación?

9) ¿Cómo se aplica el remedio y en qué cantidad?

10) ¿Después de aplicar el remedio se debe tener algún cuidado? (comida especial, baños, bebidas)

11) ¿Se puede guardar y utilizarlo en otra ocasión?

12) ¿Qué tan efectiva es la planta: tiempo del tratamiento?

13) ¿La planta se recolecta todo el año o hay periodos para hacerlo?

14) ¿Cómo la recolecta? (tiene alguna ceremonia para recolectarla)

15) ¿En qué tipo de hábitat se encuentran? (acahual, campo de cultivo, milpa, agostadero)

16) ¿Cuándo dan flores y frutos?

17) ¿Sabe si tienen machos y hembras?

18) ¿Cuál es la proporción aproximada en sexos que se encuentran? (cuántos machos y hembras encuentra).

19) ¿Se venden las plantas o los preparados? ¿Dónde se venden y quiénes las compran?

20) ¿Quién o quienes realizan principalmente las curaciones?

21) ¿El preparado puede ser usado en todo tipo de personas: hombres, mujeres, niños? (edad)

22) ¿Quiénes no deben usar las dosis?

23) ¿Usted recolecta o siembra la planta?

24) ¿Sabe si alguien la siembra?

25) ¿Dónde y cómo la siembra?

26) ¿Es fácil conseguirla?

27) ¿Le gustaría que se vendieran en el mercado?

28) ¿Quienes consumen las plantas? (gente del lugar o de fuera)

29) ¿Dónde aprendió a usarla?

30) ¿Han disminuido las poblaciones de estas plantas o son abundantes?

31) ¿Los jóvenes se interesan por lo que hace?

\section{Resultados}

Información etnobotánica. La edad promedio de los informantes fue de 73 años, con un intervalo de confianza (95\%) de \pm 27 años. El señor Eduardo Leal, de El Serrano, y la señora Asunción Parra, de Juan Rodríguez Clara, usan únicamente una planta cada uno, la mata de la víbora (Hibiscus tiliaceus L.) y el árbol de la víbora (Erythrina americana Mill.), respectivamente. Por el contrario, el señor Luciano Sánchez, residente de Sayula de Alemán, hace una mezcla de 12 plantas: guaco negro o canastilla (Aristolochia ovalifolia Duch.), camotillo (Aristolochia sp.), gorgoncillo (Piper amalago L.), redondilla (Cissampelos pareira L.), cornizuelo (Acacia cornigera [L.] Willd.), guayacán (Acosmium panamense [Benth.] Ya kovlev), zapote chico (Manilkara zapota [L.] P.Royen), hierba del cáncer (Sicydium aff. tamnifolium [Kunth] Gogn.), crespilla (Lygodium venustum Sw.), hierba del sapo (Dorstenia contrajerba L.), berenjena macho (Solanum verbascifolium L.), berenjena hembra (Solanum ochraceo-ferrugineum [Dunal] Fernald) y crucetilla (Randia aculeata L.). Este info rmante modifica el trat amiento agregando a la mezcla semillas del árbol de la 
Cuadro 2. Plantas utilizadas en la elaboración de suero anti-viperino y su forma de uso.

\begin{tabular}{|c|c|c|c|c|c|c|c|c|}
\hline $\begin{array}{l}\text { Nombre } \\
\text { científico }\end{array}$ & $\begin{array}{l}\text { No. de } \\
\text { registro* }\end{array}$ & $\begin{array}{l}\text { Nombre } \\
\text { común }\end{array}$ & Localidad & $\begin{array}{l}\text { Parte } \\
\text { utilizada }\end{array}$ & Preparación & $\begin{array}{l}\text { Dosis y tiempo } \\
\text { de tratamiento }\end{array}$ & $\begin{array}{l}\text { Cuidados } \\
\text { especiales }\end{array}$ & Restricciones \\
\hline $\begin{array}{l}\text { Hibiscus } \\
\text { tiliaceus }\end{array}$ & MRA016 & $\begin{array}{l}\text { Mata de la } \\
\text { víbora }\end{array}$ & El Serrano & Semillas & $\begin{array}{l}\text { Extracto } \\
\text { alcohólico }\end{array}$ & $\begin{array}{l}\text { Una cucharada, } \\
\text { el tiempo de } \\
\text { tratamiento es de } \\
\text { una a dos horas }\end{array}$ & Ninguno & Ninguna \\
\hline $\begin{array}{l}\text { Erythrina } \\
\text { americana }\end{array}$ & MRA017 & $\begin{array}{l}\text { Árbol de la } \\
\text { víbora }\end{array}$ & $\begin{array}{l}\text { Rodríguez y } \\
\text { Sayula }\end{array}$ & $\begin{array}{l}\text { Hojas y } \\
\text { semillas }\end{array}$ & Cocimiento & $\begin{array}{l}\text { Una taza cada } \\
\text { dos horas durante } \\
\text { seis horas }\end{array}$ & $\begin{array}{l}\text { No grasas, } \\
\text { no irritantes } \\
\text { no alcohol, } \\
\text { no corajes }\end{array}$ & Ninguna \\
\hline $\begin{array}{l}\text { Aristolochia } \\
\text { ovalifolia }\end{array}$ & MRA013 & $\begin{array}{l}\text { Guaco negro, } \\
\text { canastilla }\end{array}$ & Sayula & Raíz & Pulverización & $\begin{array}{l}30 \mathrm{~g} \text { de polvo en } \\
250 \mathrm{ml} \text { de agua } \\
\text { cada cuatro a seis } \\
\text { horas durante tres a } \\
\text { cinco días }\end{array}$ & $\begin{array}{l}\text { No grasas, } \\
\text { no irritantes } \\
\text { no agua } \\
\text { no alcohol }\end{array}$ & $\begin{array}{l}\text { No aplicar a } \\
\text { mujeres } \\
\text { embarazadas }\end{array}$ \\
\hline Aristolochia sp. & MRA003 & Camotillo & Sayula & Raíz & & & & \\
\hline Piper amalago & MRA011 & Gorgoncillo & Sayula & Raíz & & & & \\
\hline Acacia cornigera & MRA033 & Cornizuelo & Sayula & Raíz & & & & \\
\hline $\begin{array}{l}\text { Cissampelos } \\
\text { pareira }\end{array}$ & MRA019 & Redondilla & Sayula & $\begin{array}{l}\text { Planta } \\
\text { completa }\end{array}$ & & & & \\
\hline $\begin{array}{l}\text { Acosmium } \\
\text { panamense }\end{array}$ & MRA020 & Guayacán & Sayula & Corteza & & & & \\
\hline Manilkara zapota & MRA021 & Zapote chico & Sayula & Corteza & & & & \\
\hline $\begin{array}{l}\text { Sicydium aff. } \\
\text { tamnifolium }\end{array}$ & MRA008 & $\begin{array}{l}\text { Hierba del } \\
\text { cáncer }\end{array}$ & Sayula & Raíz & & & & \\
\hline $\begin{array}{l}\text { Lygodium } \\
\text { venustum }\end{array}$ & MRA022 & Crespilla & Sayula & Tallo y hojas & & & & \\
\hline $\begin{array}{l}\text { Solanum } \\
\text { ochraceo- } \\
\text { ferrugineum }\end{array}$ & MRA007 & $\begin{array}{l}\text { Berenjena } \\
\text { hembra }\end{array}$ & Sayula & $\begin{array}{l}\text { Planta } \\
\text { completa }\end{array}$ & & & & \\
\hline $\begin{array}{l}\text { Solanum } \\
\text { verbascifolium }\end{array}$ & MRA008 & $\begin{array}{l}\text { Berenjena } \\
\text { macho }\end{array}$ & Sayula & $\begin{array}{l}\text { Planta } \\
\text { completa }\end{array}$ & & & & \\
\hline Randia aculeata & MRA014 & Crucetillo & Sayula & $\begin{array}{l}\text { Planta } \\
\text { completa }\end{array}$ & & & & \\
\hline $\begin{array}{l}\text { Dorstenia } \\
\text { contrajerba }\end{array}$ & MRA012 & $\begin{array}{l}\text { Hierba } \\
\text { del sapo }\end{array}$ & Sayula & $\begin{array}{l}\text { Planta } \\
\text { completa }\end{array}$ & & & & \\
\hline
\end{tabular}

*Registro preliminar en el Herbario CIB, Instituto de Investigaciones Biológicas, Universidad Veracruzana.

víbora cuando la preparación de las 12 plantas no surte el e fecto deseado (cuadro 2).

Se encontró una fuerte correlación entre el tiempo de habitar en un lugar y el conocimiento de las plantas $(R=$ $0.98)$, aunque no hubo significancia estadística $(P=0.11)$, lo cual se debe a que el número de informantes fue pequeño. El número de plantas utilizadas podría estar relacionado con la efectividad de las mismas para tratar las mordeduras de serpientes, es decir, si una planta es muy efectiva no existe la necesidad de agregar alguna otra.

Luciano Sánchez es el único informante que lleva a cabo una ceremonia de agradecimiento al dueño del monte. En ella pide permiso para tomar las plantas y agradece que le sean proporcionadas, quema copal (Protium copal [Schltdl. et Cham.] Engl.) y dice una plegaria. Además, esta persona sólo recolecta una vez al año las plantas necesarias para el remedio antiviperino.

Partes utilizadas. La mezcla que realiza el señor Luciano Sánchez está formada por plantas completas, tallos, hojas, raíces y corteza en proporciones iguales, además de agregar a dicha mezcla, en caso necesario, siete semillas pulve- 
rizadas del árbol de la víbora (cuadro 2). Por otra parte, de la mata de la víbora se utilizan las semillas y del árbol de la víbora, las hojas.

Modo de preparación. Cada informante prepara de manera diferente el antídoto (cuadro 2). El señor Luciano Sánchez, después de recolectar las plantas, las deja secar al sol aproximadamente durante 30 días; una vez secas las pulveriza en el metate. Ya que está lista, la mezcla puede ser almacenada para emplearla después. Las semillas de la mata de la víbora (Hibiscus tiliaceus) se trituran hasta pulverizarlas y ese polvo se vierte en un litro de alcohol, pudiendo dejarse ahí para su posterior utilización. Del árbol de la víbora (Erythrina americana) se toman cinco hojas y se hierven en agua; este antiveneno no puede guardarse porque pierde su efectividad. Según Luciano Sánchez, las plantas se deben recolectar el primer viernes de marzo, ya que ese día las plantas tienen más poder para curar.

Forma de aplicación. Para todos los antídotos, la forma de aplicación es oral. La dosis única del antídoto preparado con la mata de la víbora (H. tiliaceus) es de una cucharada cafetera y el tiempo de tratamiento es de una a dos horas. Del árbol de la víbora (E. americana) se suministra una taza cada dos horas, hasta que el paciente se restablezca totalmente, lo cual ocurre normalmente en seis horas. De la mezcla de 12 plantas preparada por Luciano Sánchez se colocan $30 \mathrm{~g}$ en $250 \mathrm{~mL}$ de agua hervida; esta solución se suministra cada cuatro a seis horas, dependiendo del estado del paciente. El tratamiento dura de tres días a una semana. Adicionalmente a la administración del antiveneno, la Sra. Asunción y el Sr. Luciano practican la extracción del veneno. La primera realiza esta práctica con una aguja hipodérmica y el último la lleva a cabo con colmillos de víboras que él mismo ha capturado; antes de poder usarse, los colmillos deben ser "curados". Esto se hace colocándolos 12 días en tabaco masticado; posteriormente se sacan y se lavan con alcohol. Ambos introducen la aguja o los colmillos en las heridas causadas por la mordedura y dan masaje sobre la parte superior al lugar de la mordida.

Restricciones para la aplicación. Únicamente la mezcla de plantas preparadas por el Sr. Luciano Sánchez no debe suministrarse a las mujeres embarazadas; los demás antídotos no tienen restricciones de aplicación y pueden administrarse a todas las personas. La dosis que se aplica es la misma, sin importar el tipo de paciente de que se trate.

Cuidados especiales después de la aplicación. El antiveneno elaborado con la mata de la víbora ( $H$. tiliaceus) no requiere cuidados especiales. Sin embargo, el preparado con el árbol de la víbora (E. americana) precisa una dieta sin irritantes, sin grasas y sin alcohol, además de no hacer corajes. En el caso del antídoto obtenido por el señor
Luciano Sánchez, además de estas precauciones se debe evitar el agua (cuadro 2).

Información ecológica. Descripción de las áreas de recolección.- Los informantes de El Serrano y Rodríguez Clara tienen las plantas que utilizan en sus jardines, no así el de Sayula de Alemán. Por esta razón, los datos ecológicos se tomaron sólo de este último municipio.

Las zonas de recolección son áreas muy aisladas que se encuentran rodeadas por terrenos utilizados para agricultura y ganadería. Estas áreas son cafetales abandonados, en una etapa sucesional avanzada; una se encuentra a $80 \mathrm{~m}$ s.n.m. (zona $1 ; 18^{\circ} 54^{\prime} \mathrm{N}$ y $94^{\circ} 58^{\prime} \mathrm{O}$ ) y la otra a $100 \mathrm{~m}$ s.n.m. (zona $2{ }^{\prime} 17^{\circ} 51^{\prime} \mathrm{N}$ y $94^{\circ} 58^{\prime} \mathrm{O}$ ), y están separadas aproximadamente por $2 \mathrm{~km}$ entre sí.

Características estructurales generales.-Las áreas muestreadas presentan tres estratos: el arbóreo, el arbustivo y el herbáceo (figura 2). La riqueza florística es baja, ya que sólo se tienen entre 19 y 21 especies diferentes. En cuanto a la abundancia, los árboles con mayor frecuencia de la zona 1, Cedrela odorata L. y Croton niveus Jacq., tuvieron 23 y 5 individuos en $400 \mathrm{~m}^{2}$, respectivamente. Las especies más abundantes se encontraron en la zona 2: Alibertia edu lis (L. Rich.) A.Rich. y Casearia sp., con 16 y 13 individuos en $400 \mathrm{~m}^{2}$, respectivamente (cuadro 3).

Con base en el DAP se puede decir que los fragmentos de vegetación estudiados tienen un mayor número de individuos pequeños que intermedios y grandes, es decir, presentan una distribución en $\mathrm{J}$ invertida (figura 3 ). En la zona 1, Cedrela odorata, Tabebuia rosea (Bertol.) A.DC., Crateva aff. tapia L., Senna pendula (Willd.) H.S. Irwin et Barneby, Pithecellobium dulce (Roxb.) Benth., Trichilia havanensis Jacq. y Acacia cornigera (L.) Willd., presentan una distribución diámetrica en $\mathbf{J}$ invertida. En la zona 2 las especies que tuvieron una distribución de diámetros en $\mathbf{J}$ inve rtida fueron Alibentia edulis, Casearia sp., Citrus sinensis (L.) Osbeck, Croton niveus, Pouteria sapota (Jacq.) H.E.Moore et Stearn, Guazuma ulmifolia Lam. y Turpinia aff. occidentalis (Sw.) G.Don.

Por el contrario, en la zona 1 Coccoloba barbadensis Jacq. y Croton niveus presentaron una estructura de J normal, ya que hubo más individuos grandes (figura 4), mientras que en la zona 2 Calycophyllum candidissimum (Vahl) DC., Mangifera indica L., Bursera simaruba, el árbol 10 (sin identificar), Cedrela odorata y Tabebuia rosea tuvieron una distribución de $\mathbf{J}$ normal.

En el cuadro 4 se presentan los valores de abundancia del estrato herbáceo en las áreas muestreadas. Es importante señalar que se observó una relación directamente proporcional entre la densidad arbórea y la densidad del estrato herbáceo, es decir, si el estrato arbóreo es denso el estrato herbáceo también lo será.

\section{Discusión}



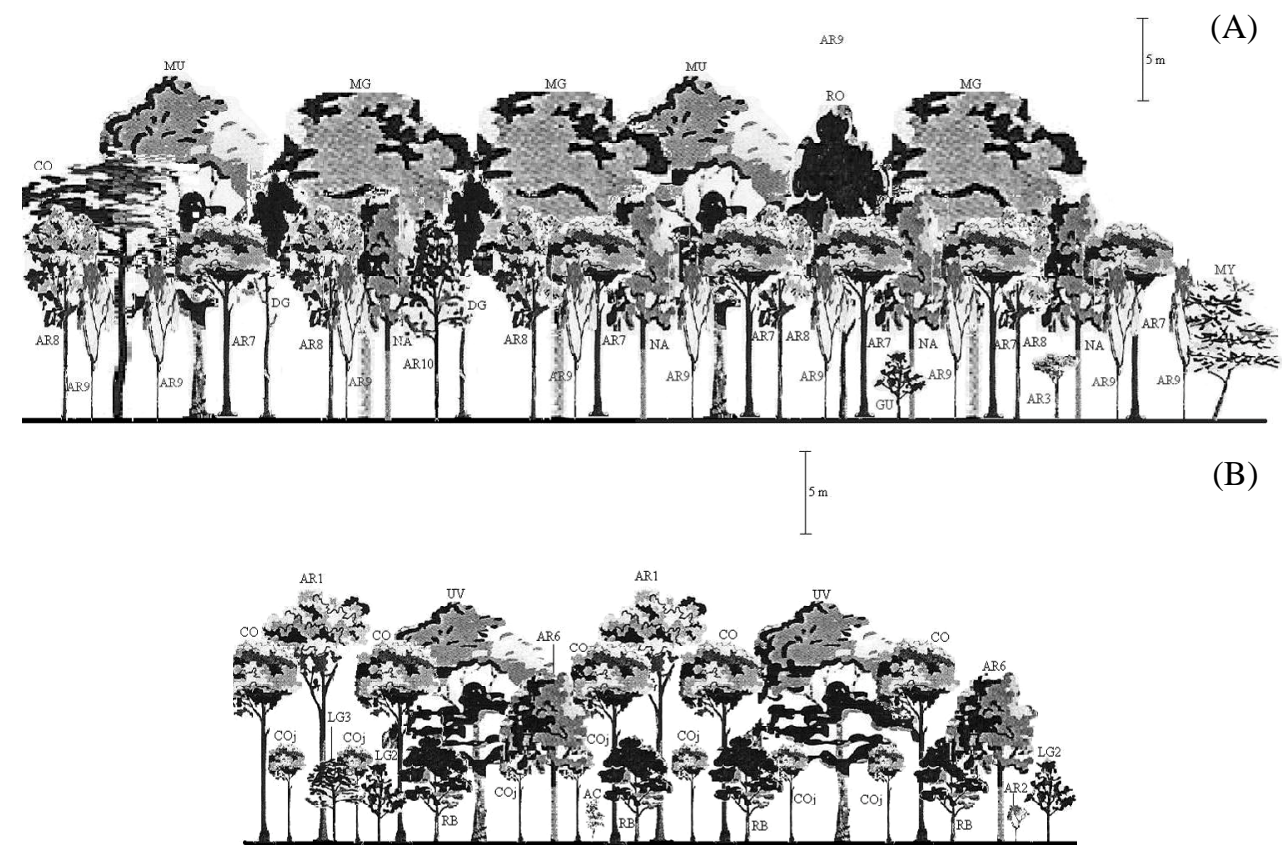

Figura 2. Perfil esquemático de la estructura de las zona 1 (A) (1854' N y 9458’ O, 80 m s.n.m., cafetal abandonado) y 2 (B) (1751' $\mathrm{N}$ y 94 58' O, 100 m s.n.m., cafetal abandonado). Los acrónimos representan. $\mathrm{AC}=$ Acacia, $\mathrm{AR} 1=$ Croton, $\mathrm{AR} 2=$ Crateva, $\mathrm{AR} 3=$ Turpinia AR6=Senna, AR7=Casearia, AR8=Árbol 8, AR9=Alibertia, AR10=Árbol 10, CO=Cedrela, COj=Cedrela joven, DG=Calycophyllum, GU=Guazuma, LG2= Pithecellobium, LG3=Trichilia, G=Mangifera, MU=Bursera, MY=Pouteria, NA=Citrus, RB y RO=Tabebuia, $\mathrm{UV}=$ Coccoloba.

Cuadro 3. Estructura del estrato arbóreo y arbustivo de las zonas $1\left(18^{\circ} 54^{\prime} \mathrm{N}\right.$ y $94^{\circ} 58^{\prime} \mathrm{O} 80 \mathrm{~m}$. s.n.m., cafetal abandonado) y $2\left(17^{\circ} 51^{\prime} \mathrm{N}\right.$ y $94^{\circ} 58^{\prime}$ O 100 m s.n.m., cafetal abandonado).

\begin{tabular}{|c|c|c|c|c|c|c|c|c|}
\hline Nombre científico & Nombre común & No. IND & A. M. (M) & $\operatorname{COB}\left(\mathrm{M}^{2}\right)$ & DE. R & DO.R. & F.R. & V. I. \\
\hline \multicolumn{9}{|l|}{ Zona 1} \\
\hline Cedrela odorata & Cedro & 23 & 13 & 38.45 & 0.5227 & 0.0375 & 0.5000 & 1.0602 \\
\hline Croton niveus & Árbol 1* & 5 & 16 & 350.15 & 0.1136 & 0.5678 & 0.1875 & 0.8689 \\
\hline Coccoloba barbadensis & Uvero & 5 & 14 & 383.41 & 0.1136 & 0.3796 & 0.2500 & 0.7433 \\
\hline Tabebuia rosea & Roble & 4 & 7 & 22.63 & 0.0909 & 0.0034 & 0.2500 & 0.3443 \\
\hline Senna pendula & Árbol 6* & 2 & 10 & 20.47 & 0.0455 & 0.0048 & 0.1250 & 0.1753 \\
\hline Pithecellobium dulce & Leguminosa $2 *$ & 2 & 5 & 18.40 & 0.0455 & 0.0010 & 0.0625 & 0.1089 \\
\hline Trichilia havanensis & Árbol 4* & 1 & 5 & 21.741 & 0.0227 & 0.0058 & 0.0625 & 0.0911 \\
\hline Acacia cornigera & Cornezuelo & 1 & 1.8 & 0.04 & 0.0227 & 0.00001 & 0.0625 & 0.0852 \\
\hline Crateva aff. tapia & Árbol 2* & 1 & 1.46 & 0.10 & 0.0227 & 0.00001 & 0.0625 & 0.0852 \\
\hline Total & & 44 & & & & & & \\
\hline \multicolumn{9}{|l|}{ Zona 2} \\
\hline Casearia sp. & Árbol 7* & 13 & 13 & 180.14 & 0.2063 & 0.0630 & 0.4375 & 0.7068 \\
\hline Mangifera indica & Mango & 6 & 19 & 394.60 & 0.0952 & 0.3179 & 0.2500 & 0.6631 \\
\hline Croton niveus & Árbol 1* & 8 & 13 & 73.24 & 0.1270 & 0.0289 & 0.500 & 0.6559 \\
\hline Alibertia edulis & Catarrita & 16 & 10 & 43.42 & 0.2540 & 0.0172 & 0.3750 & 0.6462 \\
\hline Bursera simaruba & Mulato & 3 & 20 & 155.32 & 0.0476 & 0.3667 & 0.1875 & 0.6018 \\
\hline Citrus sinensis & Naranja & 7 & 14 & 108.43 & 0.1111 & 0.0973 & 0.2500 & 0.4585 \\
\hline Calycophyllum candidissimum & Dagame & 4 & 15 & 61.02 & 0.0635 & 0.0288 & 0.2500 & 0.3423 \\
\hline Cedrela odorata & Cedro & 1 & 16 & 40.32 & 0.0159 & 0.0318 & 0.0625 & 0.1102 \\
\hline Tabebuia rosea & Roble & 1 & 18 & 21.28 & 0.0159 & 0.0189 & 0.0625 & 0.0973 \\
\hline Pouteria sapota & Mamey & 1 & 9 & 38.43 & 0.0159 & 0.0183 & 0.0625 & 0.0967 \\
\hline Sin identificar & Árbol $10^{*}$ & 1 & 13 & 24.08 & 0.0159 & 0.0104 & 0.0625 & 0.0888 \\
\hline Guazuma ulmifolia & Guácimo & 1 & 3.5 & 1.44 & 0.0159 & 0.0004 & 0.0625 & 0.0788 \\
\hline Turpinia aff. occidentalis & Árbol 3* & 1 & 3.2 & 2.08 & 0.0159 & 0.0002 & 0.0625 & 0.0786 \\
\hline Total & & 63 & & & & & & \\
\hline
\end{tabular}

*No se registraron nombres comunes para estas especies. 


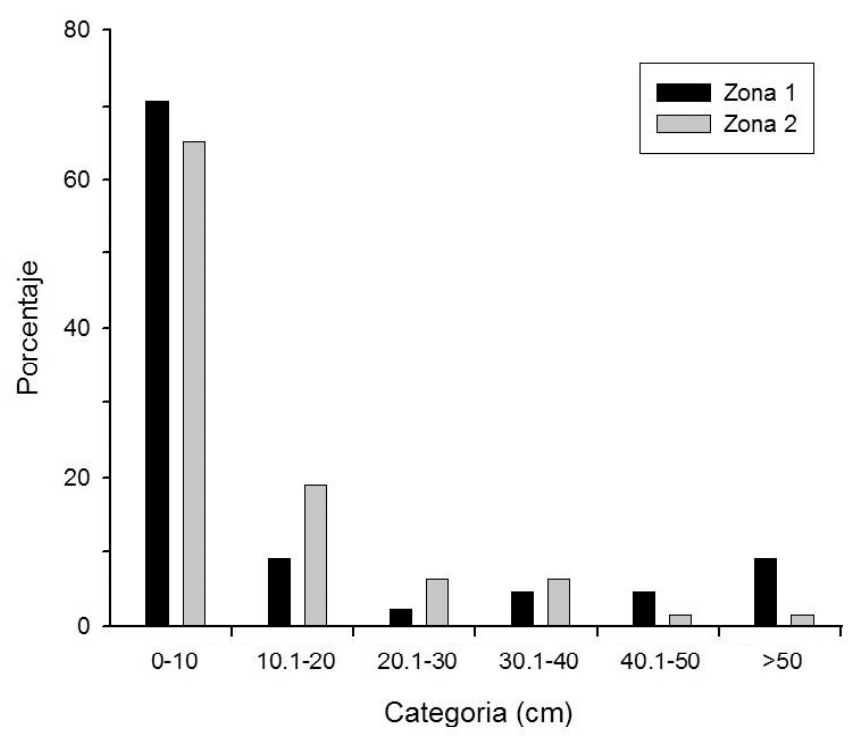

Figura 3. Estructura diamétrica de las comunidades de las zonas 1 y 2 .

Información etnobotánica. La edad de los informantes que fueron entrevistados confirma lo mencionado por Yates y Ramírez-Sosa (2004), acerca de la importancia de las personas mayores en la transmisión del conocimiento del uso de las plantas. Además, confirma la idea de que el conocimiento de las plantas está quedando en las personas mayo- res y corre el riesgo de perderse como consecuencia del reemplazo de las plantas medicinales por los fármacos sintéticos, la erosión cultural y la occidentalización de los grupos étnicos (Levy y Aguirre, 1999; Huerta, 2002). Aunado a esto, existe una tendencia reciente de las compañías multinacionales hacia la biopiratería y/o la bioprospección (Delgado-R., 2002; Choudry, 2004; Bravo, 2004; Kneen, 2004; GRAIN, 2004).

Plantas utilizadas. - Con respecto a la ceremonia realizada por el Sr. Luciano Sánchez, Rist (2000) menciona que los indígenas andinos en Bolivia y Argentina realizan ceremonias similares para pedir permiso a Dios antes de extraer las plantas medicinales. Esto y el hecho de que los demás informantes tengan las plantas en sus huertos familiares y cuiden de ellas, demuestra el profundo respeto que tienen por la naturaleza, ya que sólo toman lo que necesitan para curarse o preparar los medicamentos, y dejan individuos en pie para futuras cosechas. Esto se conoce como "gratificación diferida" y según Gómez-Pompa (2000), fue el inicio de la silvicultura.

Partes utilizadas.-Los curanderos amuzgos de Guerrero utilizan las mismas partes de las plantas reportadas en el presente estudio, pero además emplean flores, la savia y las yemas (Robinson y López, 1999). Garrido (1997) reportó el uso de hojas, tallos, cortezas y raíces de las plantas que emplean como anticrotálicos los curanderos de Catemaco, Veracruz, pero no hacen uso de las semillas ni de las plantas completas.

Cuadro 4. Densidad del estrato herbáceo de las zonas $1\left(18^{\circ} 54^{\prime} \mathrm{N}\right.$ y $94^{\circ} 58^{\prime} \mathrm{O}, 80 \mathrm{~m}$ s.n.m., cafetal abandonado) y $2\left(17^{\circ} 51^{\prime} \mathrm{N}\right.$ y $94^{\circ} 58^{\prime} \mathrm{O}$, 100 m s.n.m., cafetal abandonado).

\begin{tabular}{|c|c|c|c|}
\hline Nombre científico & Nombre común & $\begin{array}{c}\text { No. de } \\
\text { individuos }\end{array}$ & $\begin{array}{c}\text { Densidad } \\
\text { relativa }\end{array}$ \\
\hline \multicolumn{4}{|l|}{ Zona 1} \\
\hline Syngonium podophyllum & Hierba $1 *$ & 75 & 0.7732 \\
\hline Coffea arabica & Cafetos & 9 & 0.0928 \\
\hline Lygodium venustum & Crespilla & 3 & 0.0309 \\
\hline Sicydium aff. tamnifolium & Hierba del cáncer & 3 & 0.0309 \\
\hline Cyperus sp. & Hierba $2^{*}$ & 3 & 0.0309 \\
\hline Dorstenia contrajerba & Hierba del sapo & 2 & 0.0206 \\
\hline Smilax domingensis & Hierba $3 *$ & 2 & 0.0206 \\
\hline Total & & 97 & \\
\hline \multicolumn{4}{|l|}{ Zona 2} \\
\hline Syngonium podophyllum & Hierba $1 *$ & 33 & 0.6600 \\
\hline Coffea arabica & Cafetos & 9 & 0.1800 \\
\hline Sin identificar & Palma $2^{*}$ & 2 & 0.0400 \\
\hline Piper amalago & Gorgoncillo & 3 & 0.0600 \\
\hline Cyperus sp. & Hierba $2^{*}$ & 1 & 0.0200 \\
\hline Dorstenia contrajerba & Hierba del sapo & 1 & 0.0200 \\
\hline Zamia loddigesii & Palmiche & 1 & 0.0200 \\
\hline Total & & 50 & \\
\hline
\end{tabular}

\footnotetext{
*No se registraron nombres comunes para estas especies.
} 

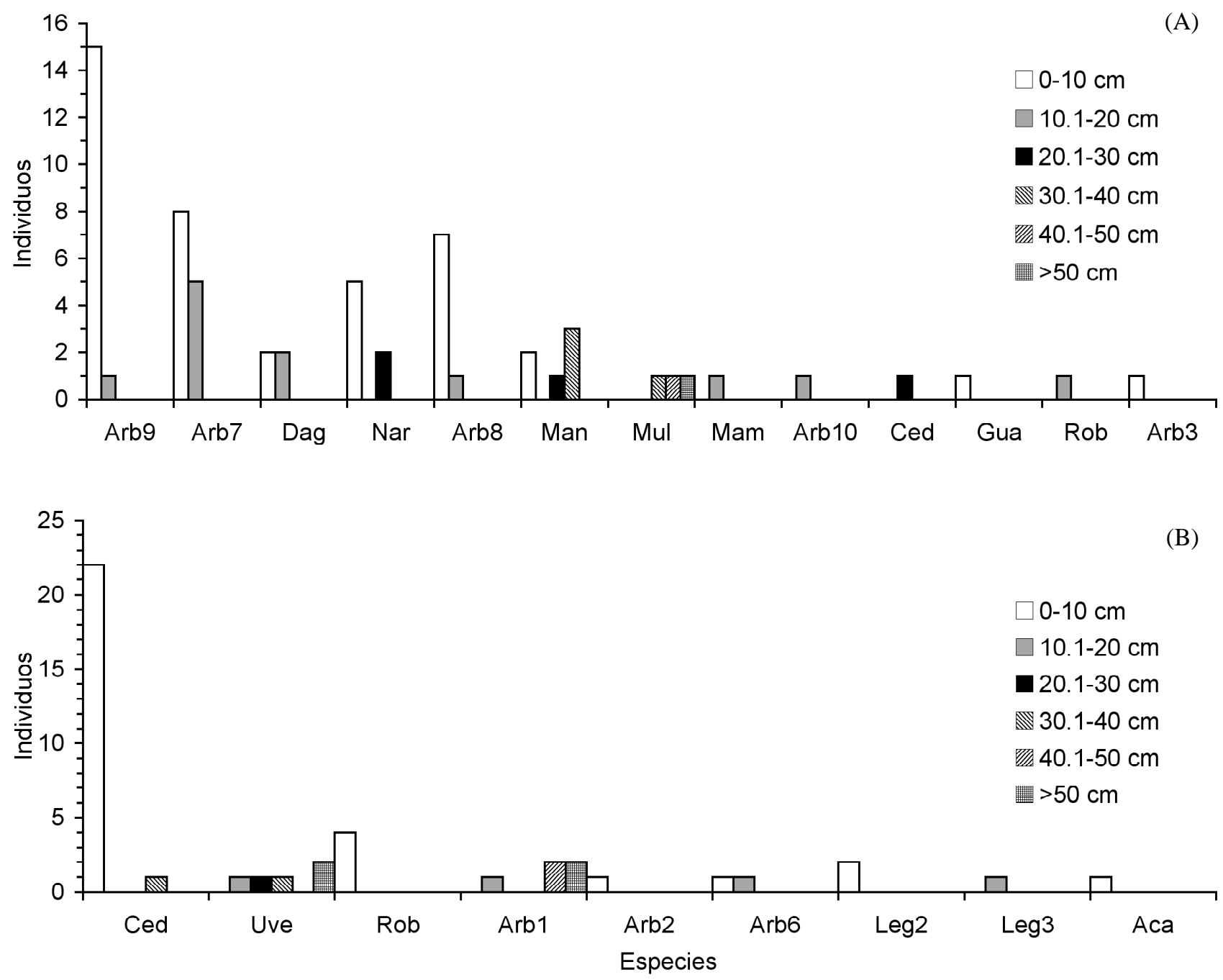

Figura 4. Estructura diamétrica por cada especie en las zonas $1(\mathrm{~A})$ y $2(\mathrm{~B}) . \mathrm{AC}=$ Acacia, $\mathrm{AR} 1=$ Croton, $\mathrm{AR} 2=$ Crateva, $\mathrm{AR} 3=$ Turpinia, AR9= Alibertia, AR6= Senna, AR7= Casearia, $\mathrm{CO}=$ Cedrela, $\mathrm{DG}=$ Calycophyllum, $\mathrm{GU}=$ Guazuma, LG2= Pithecellobium, LG3= Trichilia, $\mathrm{MG}=$ Mangifera, $\mathrm{MU}=$ Bursera, $\mathrm{MY}=$ Pouteria, $\mathrm{NA}=$ Citrus, $\mathrm{RO}=$ Tabebuia, $\mathrm{UV}=$ Coccoloba .

El uso de ciertas partes de la planta está relacionado con la observación meticulosa por parte de los informantes en un proceso milenario (Gómez-Pompa, 2000; Lewis, 2003). Esto coincide con los resultados de este estudio, ya que el conocimiento llegó a los informantes por transmisión oral a través de parientes o de curanderos.

Modo de preparación.-La fecha específica de colecta mencionada por el Sr. Luciano Sánchez coincide con lo reportado por Garrido (1997) para Catemaco, aunque dicho autor también reporta como fecha clave el 24 de junio. Una posible explicación a esto se encuentra en la fenología y la fisiología de las plantas, ya que, como en el caso de Peperomia pellucida (L.) Kunth y Passiflora incarnata L., la concentración de ingredientes activos varía de acuerdo con la fenofase e incluso cambia con respecto a la hora en que se realice la colecta (Fuentes et al., 2001; Arrigoni-
Blank et al., 2002). Por tal motivo y para encontrar una explicación más exacta desde el punto de vista biológico, se deben realizar estudios fenológicos, fisiológicos y de ingredientes activos con las plantas encontradas en este trabajo.

En lo referente a la forma de preparar los antídotos, en México hay lugares en donde no se utiliza el extracto alcohólico, como en el caso de los curanderos amuzgos de Guerrero (Robinson y López, 1999), pero sí la pulverización y el cocimiento, al igual que los informantes de Sayula y Rodríguez Clara. También hay quienes no utilizan la pulverización, como los curanderos de Aguascalientes; sin embargo, emplean ampliamente el extracto alcohólico y el cocimiento para preparar muchos remedios herbolarios (García-Regalado, 1995). En Catemaco, los curanderos utilizan el cocimiento, el machacado y el extracto alcohólico; 
además, también aplican algunas plantas frescas, como es el caso de Erythrina americana, de la cual se comen las hojas para contrarrestar el veneno de las serpientes (Garrido, 1997).

Forma de aplicación.- No es de sorprender que todos los antídotos encontrados se administren por vía oral, ya que ésta es una de las formas más comunes de suministrar los remedios herbolarios (García-Regalado, 1995; Garrido, 1997; Robinson y López, 1999; Lans et al., 2001). A diferencia de este estudio, tanto en Catemaco, Veracruz, como en Trinidad y Tobago, se ha reportado la aplicación de las plantas antiveneno por vía cutánea, en forma de emplasto, machacando las plantas y aplicándolas directamente sobre el sitio de la mordedura para reducir los efectos locales del veneno (Garrido, 1997; Lans et al., 2001).

Aunque se ha dudado de la eficacia de la ex tracción del veneno mediante la succión y el masaje, que es como lo realizan dos de los informantes, hay indicios de que es posible extraer de 22 a $50 \%$ del veneno si se realiza en los tres pri me ros minutos después de que ocurrió la mordedura; esto ayuda a disminuir los efectos tóxicos (Sanford, 1996).

Cuidados especiales después de la aplicación.- Las recomendaciones post-tratamiento registradas en el presente estudio son similares a las que hacen los curanderos de Catemaco, aunque estos últimos, además, impiden que entre una mujer embarazada al cuarto del enfermo, y recomiendan permanecer en cama durante una semana, no bañarse y no hacer esfuerzo físico (Garrido, 1997).

La recomendación de no consumir alcohol después de ser tratado con el antídoto puede tener dos explicaciones: la primera es la posibilidad de reacciones químicas con los ingredientes activos de los antídotos y la segunda tiene que ver con los efectos del alcohol sobre el paciente, como acelerar el pulso cardiaco y aumentar la presión sanguínea, lo que implica mayor circulación del veneno en el organismo del paciente (Palm Beach Herpetological Society, 2006).

Información ecológica. Características estructurales generales.-Las zonas de colecta de plantas antiviperinas presentan diferencias importantes respecto a las selvas de Los Tuxtlas. En estas últimas el estrato arbóreo presenta 66 especies, aunque las más abundantes tan sólo tienen uno y tres individuos en $400 \mathrm{~m}^{2}$ aproximadamente, Pseudolmedia sp. y Faramea occidentalis (L.) A.Rich, respectivamente (Ricker y Daly, 1998). Al comparar los V.I. de las dos zonas de estudio, se observó que los correspondientes a las especies de la zona 2 están distribuidos de manera más homogénea, no así en la zona 1, para la que los valores fueron más heterogéneos. Esto podría reflejar que la zona 2 tiene mayor diversidad que la primera, ya que una distribución homogénea de los valores de importancia puede ser un indicador de mayor equidad en la comunidad.
La distribución diamétrica en forma de $\mathrm{J}$ invertida puede reflejar la existencia abundante de micrositios favorables para el establecimiento y desarrollo inicial, si la relación tamaño-edad es estrecha y positiva; por el contrario, si la relación tamaño-edad no existe y los individuos crecen a ritmos distintos, dependiendo de la situación ambiental que los afecte, una estructura de tamaños dominada por árboles pequeños indicaría que existen muchos más sitios pobres que buenos para el crecimiento de los individuos (Martínez-Ramos y Álvarez-Buylla, 1995). Sin embargo, para establecer cuál es el caso de los sitios de colecta, deben llevarse a cabo estudios más profundos para poder plantear alternativas de manejo adecuadas, y lograr la preservación de especies como la hierba del cáncer y el guaco, que dependen en gran medida de los árboles para prosperar, ya que los necesitan como hospederos para desarrollarse. De ahí la importancia de conservar los árboles, ya que son estas especies las que crean un hábitat apropiado para las demás, evitando con ello que la comunidad se degrade (Primack, 2004). No obstante, también están las plantas que prosperan en áreas peturbadas, para las que los agroecosistemas revisten un hábitat adecuado. En este sentido, el Sr. Luciano Sánchez mencionó la creciente dificultad para encontrar las plantas necesarias para preparar su antídoto, lo que confirma que la presión ejercida por los sistemas de producción agropecuaria están eliminando las fuentes de plantas medicinales que quedan y las están aislando al fragmentar el hábitat. Esto seguramente impedirá el flujo de propágulos de una zona de diversidad a otra y terminará provocando la formación de metapoblaciones; al ocurrir este fenómeno será más difícil conservar los recursos biológicos, ya que las poblaciones aisladas tienen menor probabilidad de sobrevivir a la extinción (Akçakaya et al., 1999; Primack, 2004). Este escenario se complica si se toma en cuenta la escasez de fauna dispersora de semillas, lo que contribuye a que la vegetación relictual no pueda colonizar nuevas áreas.

Para evitar o atenuar la pérdida de diversidad biológica y cultural, se deben diversificar los agostaderos de la zona. Esto permitiría crear puentes o corredores biológicos a través de una matriz agrícola, y establecer jardines botánicos; ello posibilitará la circulación de las especies entre estas zonas y de esta manera atenuar la pérdida de la biodiversidad (Akçakaya et al., 1999; Vandermeer y Perfecto, 2007). Otra alternativa es realizar talleres acerca de plantas medicinales, su importancia biológica, económica y social, en los que participen todos los miembros de las comunidades.

Por otra parte, las plantas recolectadas para elaborar los antídotos presentan bajos valores de importancia, lo que puede ser evidencia de que son seleccionadas por su efectividad y no necesariamente por su abundancia.

Es importante destacar que en la zona estudiada el conocimiento acerca de las plantas utilizadas como antídoto para la mordedura de serpiente está en riesgo de perderse, 
y aunque no se tomaron datos para medir el desarraigo, se observó desinterés por parte de los jóvenes en aprender a utilizar esas plantas, así como un mayor uso de medicamentos sintéticos. Ambos fenómenos pueden ser los factores principales de la pérdida de dicho conocimiento. Por ello, sería importante realizar trabajos de investigación en este sentido. Aunado a lo anterior, el conocimiento de las plantas utilizadas como antídoto contra la mordedura de serpiente se concentra en personas mayores de 46 años. La ceremonia realizada por uno de los informantes y el que los demás tengan las plantas en sus jardines y cuiden de ellas es una aparente muestra de la estrecha relación que tienen con la naturaleza las personas que utilizan las plantas medicinales, ya que sólo toman lo que necesitan para curarse o preparar los medicamentos y dejan individuos en pie para poder cosechar en el futuro.

Los datos obtenidos en este trabajo son la base para realizar estudios más detallados acerca del uso de las plantas antiviperinas y las características ecológicas de los sitios de colecta que deben ser conservados en el futuro, además de resguardar el conocimiento tradicional de las plantas medicinales. Es necesario realizar ensayos farmacológicos para evaluar la efectividad de los tratamientos documentados en el presente trabajo, además de análisis fitoquímicos para aislar e identificar los ingredientes activos de las plantas utilizadas como antiviperino y relacionar ambos con la etapa fenológica de las plantas.

\section{Agradecimientos}

Agradecemos a Montserrat Gispert, Jorge A. Meave y un revisor anónimo por los atinados comentarios y observaciones al documento original.

\section{Literatura citada}

Akçakaya H.R., Burgman M.A. y Ginzburg L.R. 1999. Applied Population Ecology: Principles and Computer Exercises Using RAMAS EcoLab 2.0. 2a ed., Sinauer Associates, Sunderland, Massachusetts.

Alexiades M.N. 1996. Collecting ethnobotanical data: an introduction to basic concepts and techniques. En: Alexiades M.N. Ed. Selected Guidelines for Ethnobotanical Research: A Field Manual, pp. 53-94, The New York Botanical Garden, Nueva York.

Arrigoni-Blank M.de F., Oliveira R.L.B., Mendes S.S., Silva P.de A., Antoniolli A.R., Vilar J.C., Cavalcanti S.C.H. y Blank A.F. 2002. Seed germination, phenology, and antiedematogenic activity of Peperomia pellucida (L.) H.B.K. BMC Pharmacology 2:12.

Bravo E. 2004. Las áreas protegidas y la privatización de la vida. Biodiversidad 41:10-14.

Castro O., Gutiérrez J.M., Barrios M., Castro I., Romero M. y Umaña E. 1999. Neutralización del efecto hemorrágico inducido por veneno de Bothrops asper (Serpentes:Viperidae) por extractos de plantas tropicales. Revista de Biología Tropical
47:605-616.

Choudry A. 2004. "Conservation international": privatizando la naturaleza y saqueando la biodiversidad. Biodiversidad 40:1722.

Connell J.H. y Slatyer R.O. 1977. Mechanisms of succession in natural communities and their role in community stability and organization. American Naturalist 3:1119-1144.

Curtis J.T. 1959. The Vegetation of Wisconsin: An Ordination of Plant Communities. University of Wisconsin Press, Madison, Wisconsin.

Curtis J.T. y McIntosh R.P. 1951. An upland forest continuum in the prairie-forest border region of Wisconsin. Ecology 32:476496.

del Amo S. 1979. Plantas Medicinales del Estado de Veracruz. Instituto Nacional de Investigación sobre Recursos Bióticos, Xalapa.

Delgado-R. G.C. 2002. La Amenaza Biológica: Mitos y Falsas Promesas de la Biotecnología. Plaza y Janés, México, D.F.

Fuentes V.R.F., Méndez G., Lemes C.M., Rodríguez C.A., Soler B.A., González R. y López E. 2001. Dinámica de acumulación mensual y diaria de alcaloides y flavonoides en Passiflora incarnata L. Revista Cubana de Plantas Medicinales 3:105111.

García-Regalado G. 1995. Plantas Medicinales de Uso Tradicional en Aguascalientes. Cuadernos de Trabajo, Agricultura y Recursos Naturales No. 19. Gobierno del Estado de Aguascalientes, Aguascalientes.

Garrido A. 1997. Etnografía y análisis del uso de plantas medicinales de ocho comunidades mestizas del municipio de Catemaco, Veracruz. Tesis de Licenciatura, Facultad de Biología, Universidad Veracruzana, Xalapa.

Gispert-Cruells M. y Rodríguez-González H. 1998. Los Coras: Plantas Alimentarias y Medicinales de su Ambiente Natural. Dirección General de Culturas Populares, Instituto Nacional Indigenista, Secretaría de Medio Ambiente, Recursos Naturales y Pesca e Instituto Nacional de Ecología, México, D.F.

Gómez-Pompa A. 2000. Etnobotánica y conservación. Revista de Geografía Agrícola 31:9-15

GRAIN. 2004. ¿Buenas ideas tergiversadas? Glosario de términos relativos a los derechos. Biodiversidad 41:1-9.

Huerta C. 2002. La herbolaria: mito o realidad. <www.conabio.gob.mx/institucion/conabio_español/doctos/ huerta.htm>

INEGI [Instituto Nacional de Geografía Estadística e Informática]. 2004. Mapa digital de México. <galileo.inegi.gob.mx/website/mexico/viewer.htm> (consultado el 15 de noviembre de 2004).

Kneen B. 2004. "Redefiniendo la propiedad": sobre la propiedad privada, lo comunal y el dominio público. Biodiversidad 40:3338.

Lans C., Harper T., Georges K. y Bridgewater E. 2001. Medicinal and ethnoveterinary remedies of hunters in Trinidad. BMC Complementary and Alternative Medicine 1:10. <www.biomedcentral.com/1472-6882/1/10>

Levy S. y Aguirre J.R. 1999. Conceptualizacion etnobotánica: experiencia de un estudio en la Lacandonia. Revista de Geografía Agrícola 29:83-114.

Lewis W.H. 2003. Pharmaceutical discoveries based on ethnomedicinal plants: 1985 to 2000 and beyond. Economic Botany 57:126-134. 
Martin G.J. 2000. Etnobotánica - Manual de Métodos: Manuales de Conservación. Serie Pueblos y Plantas 1. World Wildlife Fund, Organización de las Naciones Unidas para la Educación, la Ciencia y la Cultura y Royal Botanic Gardens, Kew, NordanComunidad, Montevideo.

Martínez-Ramos M. y Álvarez-Buylla E. 1995. Ecología de poblaciones de plantas en una selva húmeda de México. Boletín de la Sociedad Botánica de México 56:121-153.

Mors B.W. 1991. Plants active against snake bite. Economic and Medicinal Plant Research 5:353-373.

Nakawa M. y Nakanishi K. 1982. Structures of cabenigrins A-I and A-2, potentent antisnake venoms. Tetrahedron Letters 23:3855-3858.

Núñez V., Otero R., Barona J., Saldarriaga M., Osorio R.G., Fonnegra R., Jiménez S.L., Díaz A. y Quintana J.C. 2004. Neutralization of the edema-forming, defibrinating and coagulant effects of Bothrops asper venom by extracts of plants used by healers in Colombia. Brazilian Journal of Medical and Biological Research 37:969-977.

Palm Beach Herpetological Society. 2006. Venomous snake bite en: <www.cdc.gov/nasd/docs/d000001-d000100/d000054/d 000054.pdf $>$ (consultado el 28 de abril de 2006).

Pereira A.N., Bettina M.R.P., Maria C.D.N., Jose P.P. y Walter B.M. 1994. Pharmacological screening of plants recommended by folk medicine as snake venom antidotes; IV. Protection against Jararaca venom by isolated constituents. Journal of Ethnopharmacolgy 60:99-100.

Primack R.B. 2004. A Primer of Conservation Biology. 3a ed. Sinauer, Sunderland, Massachussetts.

Reyes-Chilpa R., Gomez-Garibay F., Quijano L., MagosGuerrero G.A. y Ríos T. 1994. Preliminary results on the protective effect of (-) edunol, a pterocarpan from Brongniartia podalyrioides (Leguminosae), against Bothrops atrox venom in mice. Journal of Ethnopharmacolgy 42:199-203.
Ricker M. y Daly D.C. 1998. Botánica Económica en Bosques Tropicales: Principios y Métodos para su Estudio y Aprovechamiento. Diana, México, D.F.

Rist S. 2000. De la investigación a la acción: aspectos conceptuales, metodológicos e institucionales para la revalorización del saber etnobotánico en comunidades campesinas de los andes Bolivianos. Revista de Geografía Agrícola 30:7-20.

Robinson G.G. y López C.B. 1999. Patrones del Uso de Plantas Medicinales entre los Amuzgos del Estado de Guerrero, México. Instituto Lingüístico de Verano, México. <www.sil.org/mexico/amuzga/guerrero/A006e-PlantasMedicinales-aAMU.pdf> (consultado en octubre de 1999)

Ruppelt B.M., Pereira E.F.R., Gonçalves L.C. y Pereira A.N. 1991. Pharmacological screening of plants recommended by folk medicine as anti-snake venom: I Analgesic and antiinflammatory activities. Memórias do Instituto Oswaldo Cruz 86:203-205.

Sánchez-Velázquez L.R. y Pineda-López M.R. 2000. Ecología Cuantitativa de Plantas. Universidad de Guadalajara, Guadalajara.

Sanford J.P. 1996. Snake bites. En: Bennett J.C. y Plum F. Eds. Cecil Textbook of Medicine. W.B. Saunders, Philadelphia.

Shimwell D.W. 1972. The Description and Classification of Vegetation. University of Washington Press, Seattle.

Tuxill J. y Nabhan G.P. 1998. Plants and Protected Areas. A Guide to In Situ Management. Stanley Thornes, Cheltenham, Reino Unido.

Vandermeer J. y Perfecto I. 2007. The agricultural matrix and a future paradigm for conservation. Conservation Biology 21:274-277.

Yates S. y Ramírez-Sosa C.R. 2004. Ethnobotanical knowledge of Brosimum alicastrum Sw. (Moraceae) among urban and rural El Salvadorian adolescents. Economic Botany 58:72-77.

Fecha de recepción: 11 de enero de 2007

Aceptado: 6 de noviembre de 2007 